\title{
THE FLICKER RESPONSE CONTOUR FOR THE CRAYFISH, I
}

BY W. J. CROZIER AND ERNST WOLF

(From the Biological Laboratories, Barvard University, Cambridge)

(Received for publication, March 1, 1939)

I

The flicker response contour for some typical arthropods exhibits a feature of particular interest. With $A m a x^{1}$ (dragonfly, nymph) and $A p i s^{2}$ (bee) the band describing log flash intensity $(I)$ vs. flash frequency $(F)$ for threshold reaction to flicker is pronouncedly asymmetrical. This has been attributed $^{3}$ to the statistically disadvantageous position of ommatidia at the sides of the markedly convex eye of these animals. An arthropod in which this limitation does not exist because the surface of the eye is sufficiently flat ${ }^{4}$ gives a flicker response contour which is perfectly symmetrical. A symmetrical curve is also provided by the responses of vertebrates such as Pseudemys ${ }^{5}$ (turtle) and gecko ${ }^{6}$ where the excitatory elements involved constitute a single population. For these uncomplicated cases the flicker response contour $(F-\log I)$ is properly described as a probability integral. ${ }^{7}$ With most vertebrates the contour exhibits the duplex character associated with the possession of both retinal rods and cones; the asymmetry due to overlapping of the respective contributions to the $F-\log I$ curve is readily dealt with analytically. ${ }^{8}$

It is consistent with the mechanical interpretation of the $F-\log I$ asymmetry in arthropods with highly convex eyes that the curve is appropriately made more symmetrical by occluding all but the center of the optic surface. ${ }^{9}$ It is also reduced by increasing the proportion of light time to dark time in the flash cycle $;{ }^{10}$ the theory ${ }^{11}$ here calls for a greater probability

${ }^{1} J$. Gen. Physiol., 1936-37, 20, 363.

${ }^{2}$ J. Gen. Physiol., 1935-36, 19, 503; 1937-38, 21, 223.

${ }^{3} \mathrm{~J}$. Gen. Physiol., 1936-37, 20, 363; 1937-38, 21, 223.

$4 J$. Gen. Physiol., 1938-39, 22, 451.

${ }^{5}$ Proc. Nat. Acad. Sc., 1938, 24, 125, 216. J. Gen. Physiol., 1938-39, 22, 311.

'Proc. Nat. Acad. Sc., 1938, 24, 216. J. Gen. Physiol., 1938-39, 22, 55.

${ }^{7}$ Proc. Nat. Acad. Sc., 1937, 23, 71, 516. J. Gen. Physiol., 1937-38, 21, 313, etc.

${ }^{8} \mathrm{~J}$. Gen. Physiol., 1937-38, 21, 203, 313, etc.

${ }^{9} \mathrm{~J}$. Gen. Physiol., 1937-38, 21, 223.

${ }^{10} \mathrm{~J}$. Gen. Physiol., 1937-38, $21,463$.

1l J. Gen. Physiol., 1937-38, 21, 313. 
of involving marginally illuminated elements which can be concerned in excitation, although at the same time requiring a reduction in the frequency with which they can contribute to the determination of the end-point result (i.e., response), so that $F_{\text {max. }}$ is then decreased.11, 10

The crustacean $U c a$ (fiddler crab) ${ }^{12}$ and the nymph of $A$ eschna ${ }^{13}$ (dragonfly) give additional evidence of the essential asymmetry of the curve of visual effect as a function of $\log I$; the conditions of the experiments in these cases were such as probably to increase the effect. Similar indications are contained in the data on Drosophila, ${ }^{14}$ and in observations on the visual acuity and intensity discrimination of the bee. ${ }^{15}$

The asymmetry of the flicker response contour for typical large-eyed arthropods, and of the corresponding curves for visual acuity, is thus seen to be modifiable according to differences in the technic of utilizing responses to a moved system of opaque and illuminated stripes, by changes in the area of optic surface open to illumination, and by alteration of the light time fraction in the light cycle. It does not change as a function of temperature. ${ }^{16}$ The modification of asymmetry is in each instance consistent with the requirements of the view that the $F-\log I$ contour is fundamentally a symmetrical probability integral, in typical arthropods distorted by the fact that normally the intensity required for response is, at lower flash frequencies, unable to act upon the full complement of potentially excitable elements because they are not adequately oriented to receive sufficient light; the higher intensities required for higher levels of $F$ in effect increase the usable area of the retina. The formal completion of this general argument is provided by the fact that neither change of area, light time ratio, ${ }^{11}$ nor temperature" alters the symmetry of the "rod" or "cone" curves for vertebrates.

To test the strength of this interpretation has seemed desirable, since for a general theory of visual responses such as may depend upon probability considerations a valid explanation must be provided for the exceptional

12 J. Gen. Physiol., 1937-38, 21, 223 (p. 239; data in Clark, L. B., J. Gen. Physiol., 1935-36, 19, 311).

${ }^{13}$ J. Gen. Physiol., 1937-38, 21, 463 (p. 471; data in Sälzle, K., Z. vergleich. Physiol., $1932,18,347)$.

${ }^{14}$ Data in Hecht, S., and Wald, G., J. Gen. Physiol., 1933-34, 17, 517.

${ }^{15}$ Hecht, S., and Wolf, E., J. Gen. Physiol., 1928-29, 12, 727. Wolf, E., J. Gen. Physiol., 1932-33, 16, 407, 773. Wolf, E., and Crozier, W. J., J. Gen. Physiol., 1932-33, 16,$787 ; 1935-36,19,503 ; 1937-38,21,223,463$.

${ }^{16}$ J. Gen. Physiol., 1936-37, 20, 393.

${ }^{17}$ J. Gen. Physiol., 1936-37, 20, 411. Proc. Nat. Acad. Sc., 1938, 24, 216. J.Gen. Physiol., 1938-39, 22, 795. 
cases not adhering to the probability integral. Moreover, the interpretation of the visual performance curves for arthropods requires a usable interpretation of their peculiar form as contrasted with those for vertebrates. In making such a further test two points have been especially in mind:

1. It is implied that an arthropod with eye-surfaces more convex than those thus far used should exhibit in its $F-\log I$ curve a more pronounced asymmetry. By suitable reduction of the optic area it should be possible to reduce the asymmetry; an experiment of this sort is described in a following paper.

2. For Apis, Anax, and Asellus the standard deviation of $d F / d \log I$, with $F_{\text {max. }}=100$ per cent, denoted by $\sigma_{\log I}^{\prime}$ is so far as can be judged identical. 4 This, of course, refers to the upper part of the curve, beyond the intensity at which the asymmetrical "discrepancy" vanishes. Reasons already listed have shown why this function is taken to be the underlying, fundamental description of the cumulative frequency distribution of elemental excitabilities. For vertebrates this quantity is a characteristic invariant differing markedly from one kind of animal to another ${ }^{5,6,8}$ and apparently determined specifically by the relevant genetic constitution of the animal. ${ }^{18}$ For both $A n a x^{16}$ and vertebrates ${ }^{17} \sigma_{\log I}^{\prime}$ is independent of temperature and of fractional light time in the flash cycle, ${ }^{10,11}$ and for $A n a x^{9}$ (and human also) it is independent of retinal area, it is clearly an invariant of the type which rational physiological analysis requires and must seek to be in position to invoke. The quantitative agreement in $\sigma_{\log I}^{\prime}$ for Anax, Apis, and Asellus (unlooked for and unsuspected when the experiments were made) is so close as to signify a common basis in the physical organization of these arthropods. In view of other pertinent evidence this is a remarkable state of affairs, which seems unlikely to be accidental. ${ }^{4}$ It could be true that arthropods with still more highly convex eyes might show a different value of $\sigma_{\log I}^{\prime}$, particularly if the eyes are carried on independently moving stalks.

\section{II}

A form convenient for these tests is the crayfish Cambarus bartoni. The eyes are here more convex than in Anax, for example. With these animals several further types of experiment are possible. For the stock involved the data of Table I may be taken as a norm, with which (under control by check observations) the results of certain experimental treatments will subsequently be compared.

18 (In man there is some evidence to suggest that it may change slightly but systematically with age also.) 
Individuals 2.5 to $4 \mathrm{~cm}$. long were selected from a large number being raised in the laboratory. Larger individuals tend to be less consistently active. They were kept in separate dishes and fed regularly with dried shrimp and Elodea. The same 10 crayfish, with identifying numbers, were used throughout. The technic of preparation and observation was the same as that recorded in our earlier papers. After dark adaptation for several hours at $21.5^{\circ} \mathrm{C}$, threshold reaction to visual flicker was observed at this temperature, employing measurements of critical illumination in the flash at various fixed flash frequencies. The flash cycle used had equally long light and dark intervals. With slowly increasing intensity, the first definite progressive motion is taken as index of the desired end-point; movement of the antennae is too uncertain for this purpose. The reaction consists in walking movements in the direction of motion of the striped cylinder. ${ }^{19}$ This occurs very quickly when the threshold intensity has been reached. If the crayfish is at the center of its jar movements of the antennae occur first, then flexion and extension of the claws, and finally progression. The sequence of these three movements is rapid, so that with an active individual they can come at apparently the same time. If the Cambarus is at the wall of its jar and facing in the direction of the stripe motion, walking begins once threshold $I$ is reached; if facing in the opposite direction, movements of antennae and legs are the first signs of reaction, tending to push the animal backward. In some cases a crayfish in the latter position tends to turn around, toward the wall of the jar. A Cambarus oriented radially in the jar, with the head end against the wall, will occasionally show first of all a response involving merely movements of the claws, followed by turning and creeping in the direction of motion of the stripes. The measurements of critical intensity for response were chiefly based upon reactions of animals not close to the wall of the containing jar, but in fact no real differences are found when using the other modes of response described.

In view of the diurnal cycles of retinal activity found in crustaceans, ${ }^{20}$ involving movements of retinal pigment, it is to be noted that readings were taken, at several flash frequencies, during mid-afternoon hours only.

The observations are collected in Table $I$. The 10 crayfish used form a homogeneous ${ }^{19}$ lot, in the sense that the relative sensitivity of each individual is randomly distributed in time and shows no correlation in successive tests on the same day; the difference between extreme mean rank order numbers of 2 individuals $=3.1 \times \mathrm{P} . \mathrm{E}_{\text {.diff. }}$. The dispersions of the critical intensities are of the same order as found in Asellus, ${ }^{4}$ but are a little smaller. The scatter of the determinations of critical intensity is directly proportional to the mean (Fig. 1).

III

The $F-\log I_{m}$ curve for Cambarus is markedly asymmetric (Fig. 2). The relative distortion of the curve is greater than with bee and dragonfly

19 J. Gen. Physiol., 1936-37, 20, 211, 363-393; 1937-38, 21, 17, etc.

20 Welsh, J. H., Proc. Nat. Acad. Sc., 1930, 16, 386. Biol. Bull., 1936, 70, 217. Under conditions of continued darkness diurnal migrations of retinal pigment occur in Cambarus also, as Dr. Welsh has kindly informed us on the basis of his recent work. 
nymph (Fig. 3). This was expected (section I) on the basis of the greater curvature of the retinal surface of the crayfish.

On a probability grid (Fig. 3) the sharp breaking of the curve at $\log I_{m}$ $=c a . \overline{1} .1$ separates the function into two parts: below this level there is wide departure from the course of the curve fitting the upper portion of the data. The departure is of exactly the same kind as with bee and dragonfly larva. The agreement in the general form of the distorted part of the curve

TABLE I

Mean critical flash intensities, with the probable errors of the dispersions, as $\log I_{m}$ and $\log$ P.E.1.1 ${ }_{11_{1}}$, millilamberts for various flash frequencies ( $F$ per sec.), for Cambarus bartoni. Flash cycle with equally long dark and light period, i.e. $t_{L} / t_{D}=1$; temperature $=21.5^{\circ}$. At each $F$, three observations on each of the same 10 individuals used at all points. The individuals are shown to be an essentially homogeneous group (see text).

\begin{tabular}{|c|c|c|}
\hline$F$ & $\log I_{m}$ & $\log$ P.E. $\mathbf{E}_{\mathbf{I}_{\boldsymbol{I}_{1}}}$ \\
\hline 2 & $\overline{4} .4955$ & $\overline{5} .0785$ \\
\hline 3 & $\overline{3} .0976$ & $\overline{5} .2420$ \\
\hline B & $\overline{\mathbf{3}} .6017$ & $\overline{5} .6766$ \\
\hline 7 & $\overline{\mathbf{3}} .8898$ & $\overline{4} .3462$ \\
\hline 10 & $\overline{2} .1483$ & $\overline{4} .5181$ \\
\hline 16 & $\overline{\mathbf{2}} .3993$ & $\overline{3} .0302$ \\
\hline & $\overline{\mathbf{2}} .3568$ & $\overline{4} .3508$ \\
\hline 20 & $\underline{\mathbf{2}} .6851$ & $\overline{4} .9976$ \\
\hline & $\overline{2} .6816$ & $\overline{4} .8320$ \\
\hline 26 & $\overline{2} .7744$ & $\overline{3} .2862$ \\
\hline 30 & $\overline{\mathbf{2}} .9442$ & $\overline{4} .9294$ \\
\hline 36 & 1. 1014 & $\overline{3} .1486$ \\
\hline 40 & $\overline{1} .3564$ & $\overline{3} .8599$ \\
\hline 43 & 1. 6424 & $\overline{3} .9375$ \\
\hline 46 & $\overline{\mathbf{1}} .9352$ & $\overline{2} .2167$ \\
\hline 47 & 0.2997 & $\overline{2} .7560$ \\
\hline 48 & 0.6280 & $\overline{1} .0103$ \\
\hline 49 & 0.8756 & $\overline{1} .0178$ \\
\hline 50 & 1.6442 & 0.2579 \\
\hline
\end{tabular}

(Fig. 4) can scarcely be fortuitous. It finds a rational explanation in the increasing probability of the involvement of marginal ommatidia as the critical intensity is increased through elevation of $F$; the disadvantage of the marginal ommatidia is due to the mechanical conditions affecting their reception of light, and not to an intrinsically lower excitability. ${ }^{\circ}$

The slope of the fundamental curve (Fig. 3) is measured by $\sigma^{\prime}{ }_{\log I}$, the standard deviation of the frequency distribution $d F / d \log I$ vs. $\log I$ (with 


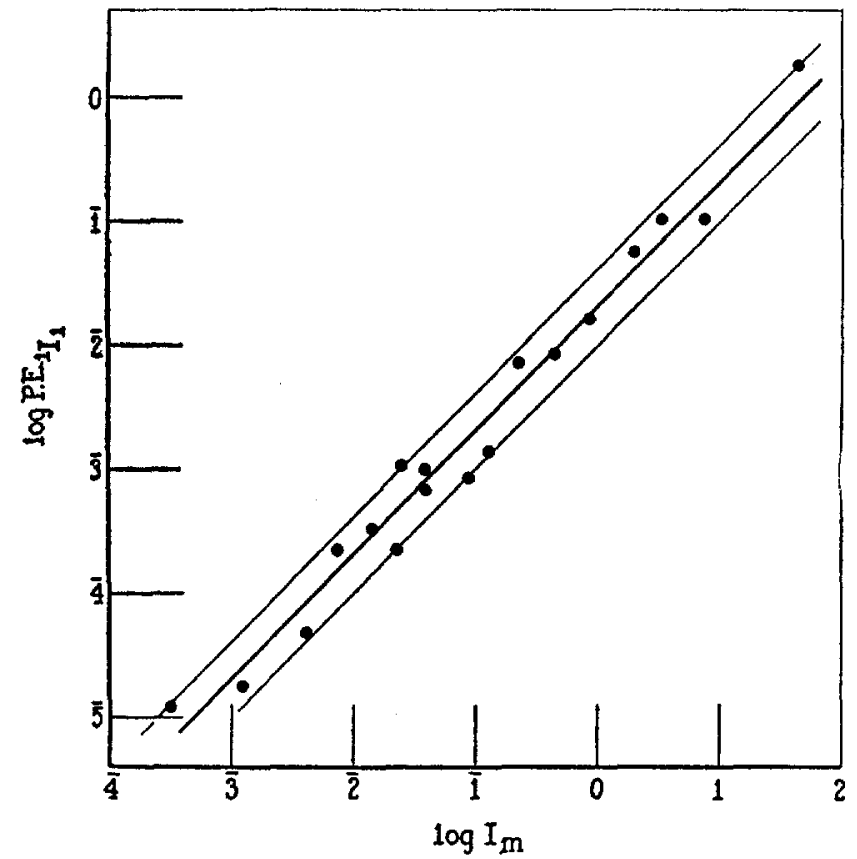

FIG. 1. The dispersion (P.E. III $_{1}$ ) of the determinations of critical intensity for reaction of Cambarus to visual flicker is directly proportional to the mean critical intensity. Data in Table I.

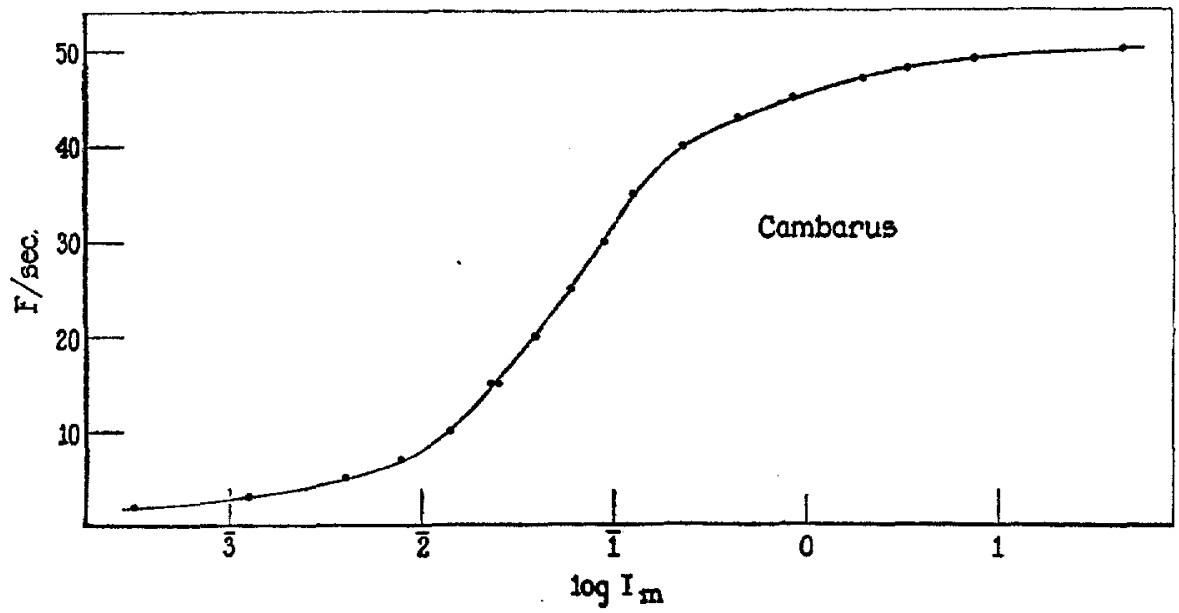

Fig. 2. The sigmoid relation between $\log I_{m}$ and $F$ for Cambarus (Table I). The curve is not symmetrical (see Fig. 3). 
$F_{\max .}=100$ per cent). The fact has been commented upon 4 that this quantity is the same for such different animals as bee, dragonfly nymph, and isopod. For Cambarus it is clearly much greater (Fig. 3). This signifies that the frequency distribution for the excitability thresholds of the neural elements concerned is more widely spread. It may be entertained as a suggestive possibility that the great curvature of the optic surface, perhaps combined with the position of the eyes upon independently movable stalks as contrasted with the condition in Anax, Asellus, Apis, contributes in a

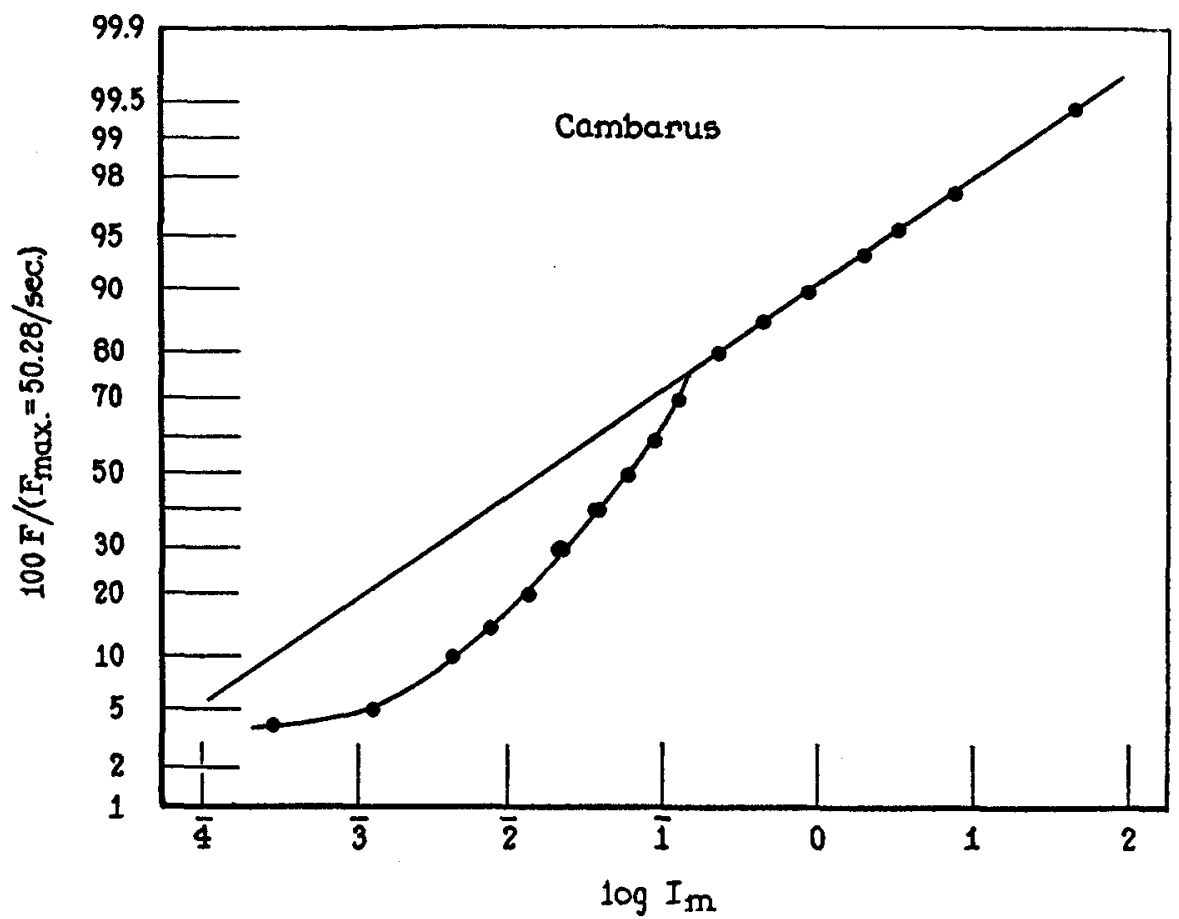

FIG. 3. The $F-\log I_{m}$ data for Cambarus (Fig. 2) shown upon a probability grid. The departure below ca. 74 per cent of $F_{\max }$, is discussed in the text.

decisive way to a statistically greater spread of the curve by increasing the possibility of moment-to-moment fluctuation in the effective thresholds of individual ommatidia.

These considerations apply to $\sigma_{\log 1}^{\prime}$, and for different animals it is to be noted that there is no correlation to be found between the absolute values of $F_{\max }$ and other properties of the flicker response contour. For Anax, under the same conditions of observation, $F_{\max .}=60.9$, for Cambarus, 50.3 ; yet the "break" in the $F-\log I$ curve comes at precisely the same 
level of intensity and of $100 \mathrm{~F} / F_{\max .}$ (Fig. 3); for $A$ pis (by a slightly different method of observation) $)^{2}$ the break comes at precisely the same relative level of $F$ (Fig. 3).

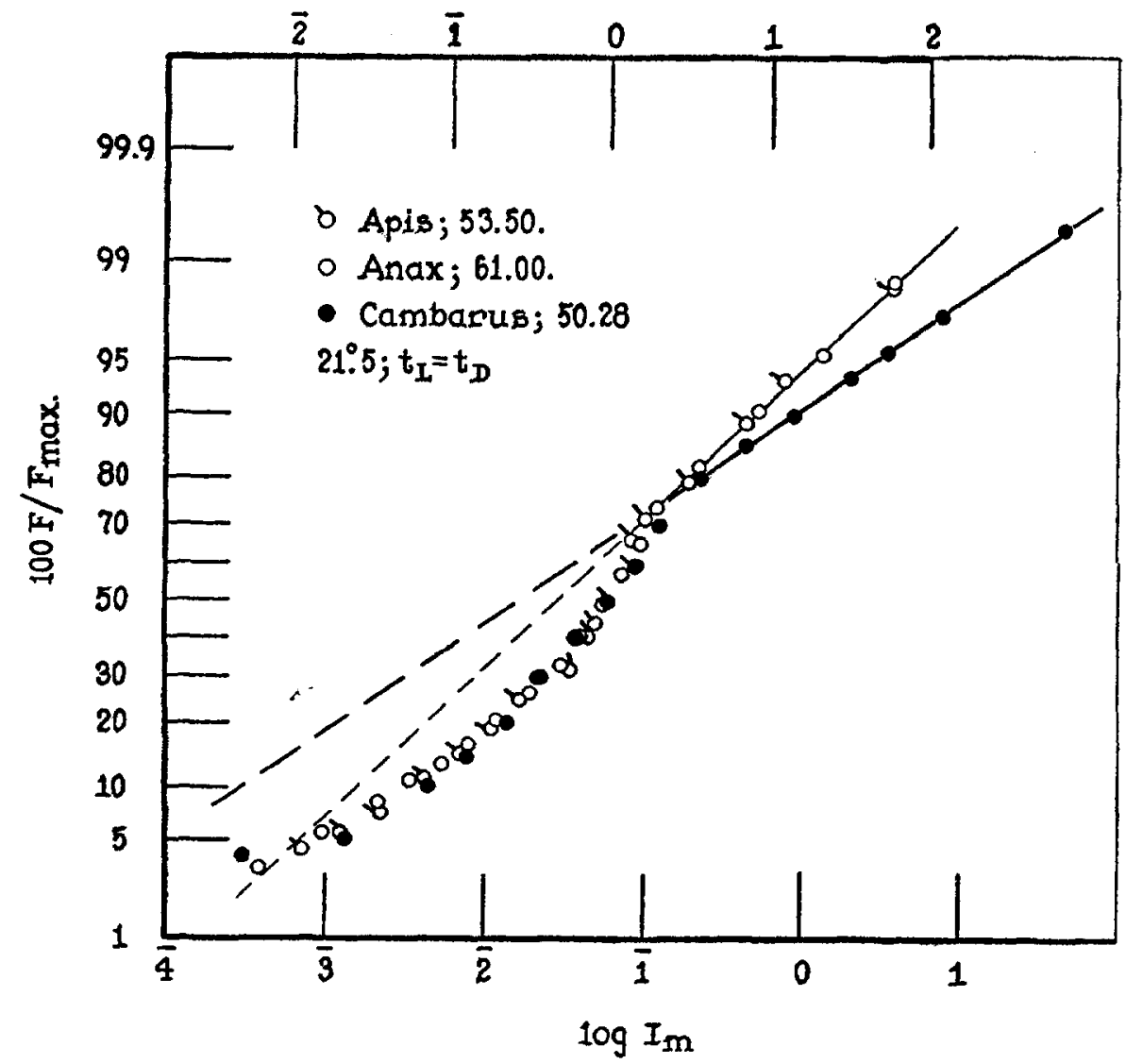

FIG. 4. The slope of the probability integral expressing the "fundamental curve" for Cambarus is less than for Apis and Anax and Asellus; the form of the curve for Anax and $A$ pis is essentially the same, but separated by 1.22 units on the $\log I$ axis; this slope is identical with that obtained for the uncomplicated curve given by the comparatively flat-eyed Asellus. ${ }^{4}$ The shape of the discrepant parts of the three curves, discussed in the text, is very nearly the same.

In terms of the analysis we are engaged upon, $F_{\max }$. is to be regarded as a measure of the total number of neural effect units capable of involvement in the discrimination of flicker. ${ }^{21}$ The curious fact that (with $t_{L} / t_{D}=1$ ) $F_{\max .}$ is for a great variety of forms (dark adapted) confined to a compara-

${ }^{21}$ J. Gen. Physiol., 1937-38, 21, 17. 
tively very narrow range of values $(=48$ to 63 ) has been commented on;22 however, in one animal (gecko $0^{6}$ ) this parameter is as low as 27 per second. The properties of the curves for different forms can be compared, however, only after reduction to a percentage scale $\left(F_{\max .}=100\right)$. For sundry convex-eyed arthropods upon which data are available-Apis, ${ }^{9,15} A$ nax, 9 $U c a,{ }^{9}$ Drosophila ${ }^{14}$-as well as for the present data on Cambarus, the rate of increase of the slope $d F / d \log I$ increases up to $F=c a$. 70-74 per cent of $F_{\text {max. }}$ (cf. Fig. 3). This fact of itself suggests that a common physical factor is responsible for the peculiar form of the curves. The deduction is reinforced by the consideration that (Fig. 4) the form of the "discrepant" portion of the $F-\log I$ curve, responsible for the asymmetry of the contour as a whole, is remarkably similar. If the common physical factor is taken to be the convexity of the optic surface (and consequent angular divergence of the ommatidial axes) the congruence of the (non-specific) forms of the "discrepant" portions of the several curves in Fig. 4 is readily accounted for. The form of the $F-\log I$ curve in the region of lower critical intensities is thus explained by the fact that, $F$ being made greater, a higher critical $I$ is necessitated, which makes possible the involvement of a larger number of receptor units-the effective area of the eye is therefore increased-and the relative rate of increase of critical intensity per unit increase of $F$ is therefore less than if the factor of ommatidial angular divergence were not involved.

The position can be further checked by investigating the effect of internally isolating the ommatidia by utilizing the migrations of retinal pigment induced by hormones acting upon crustacean chromatophores, as discussed in a succeeding paper. ${ }^{23}$

The final fact to be recognized is that in these data the value of $\sigma_{\log I}^{\prime}$ for the "fundamental curve" (cf. Fig. 3) is definitely greater than for Apis and Anax, and than that for Asellus (no asymmetry). The statistical conception of the involvement of excitation elements in the determination of response rather distinctly calls for this finding in the present case. The optic surfaces of Cambarus are not only more convex, but the two eyes are independently movable. A wider variety of chances is thereby presented for the photic excitation of the various peripheral units. This implies a higher value of $\sigma_{\log I}^{\prime}$, such as Fig. 3 demonstrates. The conclusion can be checked by measurements with other crustaceans. ${ }^{24}$

22 J. Gen. Physiol., 1936-37, 20, 393.

${ }^{23}$ Crozier, W. J., and Wolf, E., 1939, Biol. Bull., 77, 126.

24 The visual acuity data with the fiddler crab Uca (Clark, L. B., J. Gen. Physiol., $1935-36,19,311$ ) were obtained by means of tests with a single moving stripe. This 
We are indebted to Dr. Gertrud Zerrahn-Wolf for assistance in connection with the observations.

\section{IV \\ SUMMARY}

The $F-\log I$ curve for threshold response to visual flicker has been determined for the crayfish Cambarus bartoni. As predicted on the basis of the higher curvature of the optic surface, the flicker response contour is more asymmetrical than for bee and dragonfly nymph under comparable conditions of temperature and light time fraction of flash cycle. The mechanical origin of this asymmetry is thus confirmed, and is further supported by the similar forms of the $F-\log I$ curves in bee, dragonfly larva, and crayfish in the lower portion of the curves (up to $F=70$ per cent $F_{\max }$ ). The slope of the fundamental curve for crayfish, deduced by analysis of the data, is lower than for bee, dragonfly nymph, or Asellus. This signifies a wider spread of the effective distribution of elemental $\log I$ thresholds involvable in the response to flicker, and may be traced either to the greater curvature of the eye-surfaces or to their position upon movable pedicles. The results are therefore consistent with the statistical conception of the nature of effects recognizable as due to the activity of excitable elements.

inevitably implies, from the standpoint herein used, a steeper ${ }^{12} \log I$ curve-since a smaller number of excitation units will be produced by movement of a single stripe than by the use of a series of them. The importance of the number of light-dark transitions has been commented upon in other connections. ${ }^{15}$ Similarly, in tests of visual acuity $^{15,21}$ the striped plates are moved slowly. This again implies a low number of light-dark excitations as compared with the situation in tests for recognition of (response to) flicker. A smaller number of excitation units implies lesser relative variability, and consequently a smaller value of $\sigma_{\log l}^{\prime}$, as is found for the bee. ${ }^{2}$ The decisive comparisons of such data require further experiments with forms such as $U c a$, which we expect to make shortly. 